\title{
Optimizing perception: Attended and ignored stimuli create opposing perceptual biases
}

\author{
Mohsen Rafiei $^{1} \cdot$ Sabrina Hansmann-Roth ${ }^{1} \cdot$ David Whitney $^{3} \cdot$ Árni Kristjánsson $^{1,4} \cdot$ Andrey Chetverikov $^{2,5}$ \\ Published online: 24 April 2020 \\ (C) The Psychonomic Society, Inc. 2020
}

\begin{abstract}
Humans have remarkable abilities to construct a stable visual world from continuously changing input. There is increasing evidence that momentary visual input blends with previous input to preserve perceptual continuity. Most studies have shown that such influences can be traced to characteristics of the attended object at a given moment. Little is known about the role of ignored stimuli in creating this continuity. This is important since while some input is selected for processing, other input must be actively ignored for efficient selection of the task-relevant stimuli. We asked whether attended targets and actively ignored distractor stimuli in an odd-one-out search task would bias observers' perception differently. Our observers searched for an oddly oriented line among distractors and were occasionally asked to report the orientation of the last visual search target they saw in an adjustment task. Our results show that at least two opposite biases from past stimuli influence current perception: A positive bias caused by serial dependence pulls perception of the target toward the previous target features, while a negative bias induced by the to-be-ignored distractor features pushes perception of the target away from the distractor distribution. Our results suggest that to-be-ignored items produce a perceptual bias that acts in parallel with other biases induced by attended items to optimize perception. Our results are the first to demonstrate how actively ignored information facilitates continuity in visual perception.
\end{abstract}

Keywords Feature distribution learning $\cdot$ Serial dependence $\cdot$ Attention $\cdot$ Visual search $\cdot$ Perception

Public Significance Statement: We used visual search to show that both attended and ignored items in a visual search task contribute to the optimization of visual perception. Previously attended items create an attractive bias (serial dependence) while ignored items produce a repulsive bias in the perception of a search target.

Electronic supplementary material The online version of this article (https://doi.org/10.3758/s13414-020-02030-1) contains supplementary material, which is available to authorized users.

Mohsen Rafiei

mor4@hi.is

1 Faculty of Psychology, University of Iceland, Reykjavik, Iceland

2 Donders Institute for Brain, Cognition and Behavior, Radboud University, Nijmegen, the Netherlands

3 Department of Psychology, The University of California, Berkeley, CA, USA

4 School of Psychology, National Research University, Higher School of Economics, Moscow, Russian Federation

5 Cognitive Research Lab, Russian Academy of National Economy and Public Administration, Moscow, Russia

\section{Introduction}

Imagine searching for an apartment for your dream vacation. After looking at a throng of ramshackle flats that are little more than distractions, even a half-decent room would look nice. However, when you see a few places that match your target criteria, each one you look at affects how the next one is perceived. Such contextual and sequential presentation effects are ubiquitous in social psychology (Simonsohn \& Loewenstein, 2006; Simonson \& Tversky, 1992). But can what we look for be affected by distractors and previously seen targets in visual search within ensembles of visual stimuli?

Perception is noisy and ambiguous, both due to external noise (e.g., differences in illumination, blur, and occlusion) and due to internal noise in the brain. The visual system might therefore utilize multiple sources of information to make correct inferences in the presence of noise. For example, knowledge of the statistics in natural images can help in perceptual decisions about visual ensembles, such as about orientation (Girshick, Landy, \& Simoncelli, 2011), motion speed (Sotiropoulos, Seitz, \& Seriès, 2011; Weiss, Simoncelli, \& Adelson, 2002), or the color of objects (Allred, 2012; 
Brainard, 2009). The same knowledge, however, leads to biases in perception - for example, perceived orientation is biased towards cardinals (Girshick et al., 2011; Wei \& Stocker, 2017). Similarly, knowledge that the visual input is mostly constant over time might help to optimize perception in the real world (van Bergen \& Jehee, 2019), but leads to biases from recently seen stimuli in the lab - an effect coined serial dependence by Fischer and Whitney (2014).

Here we ask if the visual system utilizes multiple sources of information to optimize perception of visual ensembles such as when we search for targets among distractors. A search task is particularly interesting since it involves ensembles that involve attended stimuli (targets) and to-be-ignored stimuli (distractors). For example, a radiologist might look for signs of tumor on an $\mathrm{X}$-ray image while ignoring salient distractors, such as bones. While many studies have demonstrated how attended items create perceptual biases (see below), the potential role of tobe-ignored items has not been addressed in the same way. Natural environments often involve situations involving distracting stimuli that need to be actively ignored rather than simply not attended. We may need to select targets that meet our goals, that may be hard to distinguish from others, and that must then be actively rejected. Active inhibition of irrelevant items is observed for example during visual search (Arita, Carlisle, \& Woodman, 2012; Beck \& Hollingworth, 2015; Cunningham \& Egeth, 2016). The biases created by such to-be-ignored items have not been studied to the same degree as target-based effects (but see Chelazzi, Marini, Pascucci, \& Turatto, 2019; Gaspelin, \& Luck, 2018; Geng, Won, \& Carlisle, 2019; Noonan, Crittenden, Jensen, \& Stokes, 2018).

In the context of visual search, previous studies have assessed biases in templates that observers use for search. Geng and colleagues (Geng, DiQuattro, \& Helm, 2017; Geng \& Witkowski, 2019; Won \& Geng, 2018; Yu \& Geng, 2019) showed that the target template, that is, the representation of the target used for search assessed through analysis of search times for different targets, can be gradually biased away from distractors. Due to noisy and unstable visual input (e.g., because of occlusions or eye movements as well as noise inherent in the nervous system), the potential function of such biases from ignored distractors could be to generate a predictive code in order to correct possible errors and to stabilize perception. However, it is not clear to what extent such effects might cause biases in target perception in visual search.

The effect of distractors on perception might be especially strong when the distractor representation can be used to facilitate search on following trials. We have previously shown that the visual system can implicitly learn the feature distributions of a set of to-be-ignored items (Chetverikov, Campana, \& Kristjánsson, 2016, 2017b, c, d, 2020; Hansmann-Roth, Chetverikov, \& Kristjánsson, 2019; for review see Chetverikov, Hansmann-Roth, Tanrikulu, \& Kristjánsson, 2019, and Chetverikov, Campana, \& Kristjánsson, 2017a).
Our feature distribution learning (FDL) paradigm shows that observers learn remarkably intricate details of distributions of distractor features, not only their mean and variance, but the probability distributions of the distractors, be it a Gaussian, uniform, skewed, or bimodal distribution (Chetverikov et al., 2016). The particular kind of a search task utilized in these studies - an odd-one-out search - ensures that observers have to analyze both target and distractors, because otherwise the target identity cannot be determined. While the target-defining feature is not known in advance, the target can often be easily found because of the similarities among the distractors. In addition, the distractor distribution remains constant for a few trials. Observers are therefore implicitly prompted to encode the distractors to facilitate search, making this an ideal task to test whether representations of ignored items bias perception.

In addition to currently present distractors, information about previous targets can also help with identifying the current target. Fischer and Whitney (2014) found that the judgment of the orientation of a Gabor patch can be strongly biased towards the previously perceived Gabors (see Kiyonaga, Scimeca, Bliss, \& Whitney, 2017, for review). Such serial dependence has been shown to occur for stimulus dimensions as varied as shape (Manassi, Kristjánsson, \& Whitney, 2019), position (Bliss, Sun, \& D'Esposito, 2017; Manassi, Liberman, Kosovicheva, Zhang, \& Whitney, 2018), eye gaze (Alais, Kong, Palmer, \& Clifford, 2018), body size (Alexi et al., 2018), or perceived motion coherence (Suarez-Pinilla, Seth, \& Roseboom, 2018). Fischer and Whitney (2014) found that serial dependence was produced by attended items only and suggested that attention serves as a "gating" mechanism for serial dependence (see also Fornaciai \& Park, 2018; Fritsche \& de Lange, 2019). We therefore expect that previously attended items will bias the perception of the current target in the context of visual search as well, further optimizing target perception.

In sum, our aim was to study the simultaneous effects of previously attended (targets) and ignored (distractors) items on perceived orientation of a line presented in isolation. After searching for an odd-one-out line among distractors for several trials, observers were presented with a single line and were asked to adjust its orientation to the orientation of the target seen on the last trial. Given that targets on consecutive trials varied, we were able to measure any serial dependence from preceding targets. But importantly, we additionally assessed whether the to-be-ignored items can also cause a bias in the line-orientation judgments.

\section{Method}

\section{Participants}

Twenty participants $(11$ females and nine males, mean age $=$ 31.55 years) participated in the experiment. They signed a 
consent form that included a brief description of the experimental procedure. Each test took about $1 \mathrm{~h}$. All participants had normal or corrected-to-normal vision.

\section{Stimuli and procedure}

The design of the experiment is shown in Fig. 1. All stimuli were presented on a gray background on a 24 -in. Asus monitor with a $1,920 \times 1,080$-pixel resolution at a viewing distance of approximately $70 \mathrm{~cm}$. MATLAB (2016) with Psychtoolbox-3 (Brainard, 1997; Kleiner, Brainard, \& Pelli, 2007) was used to program and run the experiment.

The experiment contained 264 blocks. Each block had four to five search trials (1,188 trials per participant) followed by an adjustment task. On search trials (following the design of Chetverikov et al., 2016), participants searched for an oddly oriented line in an array of 36 lines displayed in a $6 \times 6$ matrix at the center of the screen. The length of each line was $1^{\circ}$. The positions of the lines on the screen were jittered by randomly adding values between $\pm 0.5^{\circ}$ to both the vertical and the horizontal coordinates. Participants were asked to press the E key when the target (an oddly oriented line) was among the three upper rows and press the D key when the target was located in the lower three rows. If the response was incorrect, the word "Error" appeared for $1 \mathrm{~s}$ on the screen. To encourage participants to be as fast and accurate as possible we used a scoring system based on the formula: Score $=10+(1-\mathrm{RT}) * 10$, where RT is the response time in seconds, while for errors: Score $=-\mid 10+$
$(1-\mathrm{RT}) * 10 \mid-10$. This equation results in positive scores for correct responses faster than $2 \mathrm{~s}$ and negative scores otherwise. The score for each trial was shown on the screen following each response.

The orientations of the distractors on learning trials were drawn from either a truncated Gaussian distribution with a standard deviation of $15^{\circ}$ or from a uniform distribution with a range of $60^{\circ}$. The mean and the type of the distribution were kept constant within a block. The orientation of the targets on each trial was selected pseudo-randomly. On the last trial in each block (i.e., series of learning trials, trial $N$ ), the target orientation was selected from a range of $-70^{\circ}$ to $+70^{\circ}$ distance to the distractor distribution mean in $4^{\circ}$ steps, so that targets were clockwise $(\mathrm{T}>\mathrm{D})$ relative to the distractor mean on half of the trials and counter-clockwise $(\mathrm{T}<\mathrm{D})$ on the other half. The distances in orientation space between target and distractor mean on trial $N$ were counterbalanced with the distances between the target on trial $N$ and target on trial N-1. To this end, on trial $N-1$ the target had either a $+10^{\circ}(\mathrm{T}>\operatorname{PrevT})$ or $-10^{\circ}(\mathrm{T}<\mathrm{prevT})$ distance from the target on trial $N$. On the rest of the learning trials in each block, targets were oriented 60 $120^{\circ}$ away from the mean of the distractor distribution.

At the end of each block (after the last trial $N$ ), participants were asked to match the orientation of a single test line to the target orientation on trial $N$. The initial orientation of the test line was selected randomly. The test line was always presented at the center of the display. Participants were encouraged to respond as quickly and accurately as possible. The response time was limited to $6 \mathrm{~s}$.

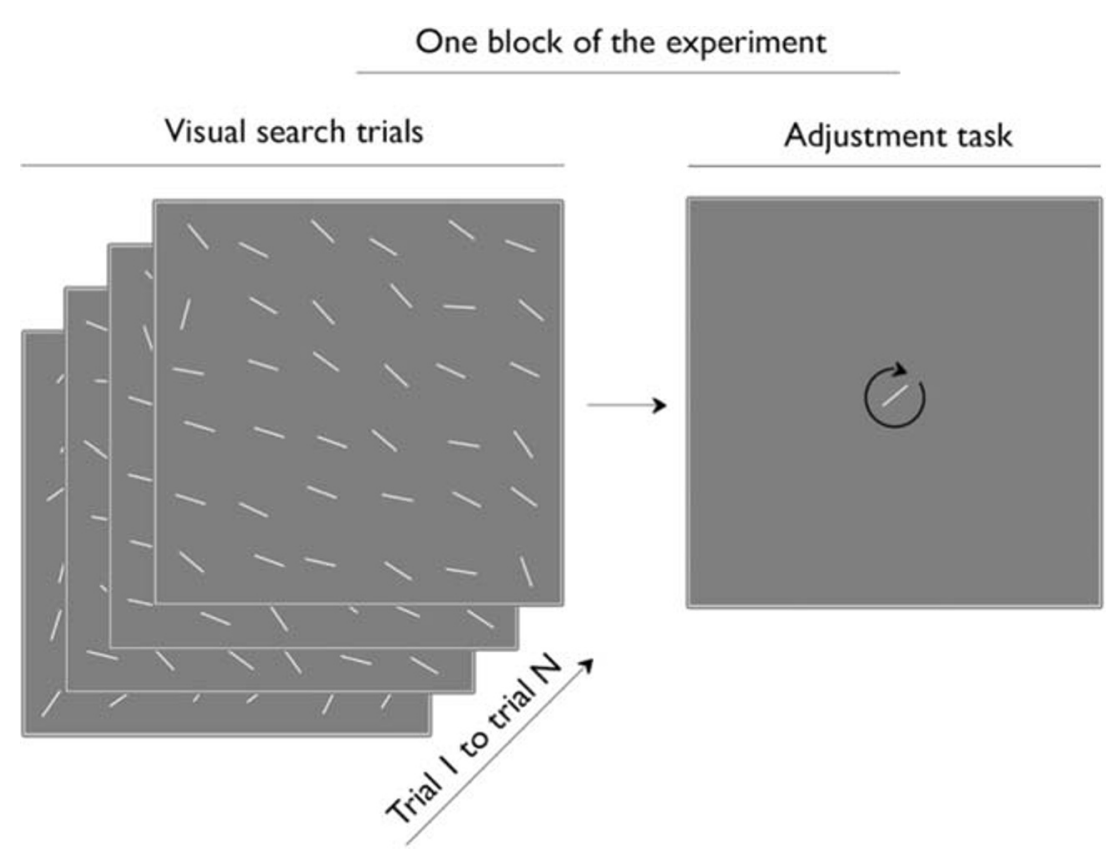

Fig. 1 Design of the experiment. In each block, participants were asked to perform a visual search task that had four to five trials (learning trials); they searched for an oddly oriented line in a search array of 36 lines shown in a $6 \times 6$ matrix in the middle of the screen, and subsequently they had to adjust the orientation of a single randomly oriented line to the orientation of the target that was presented on trial $N$ 


\section{Data analysis}

To filter out trials with exceedingly long response times, trials with log-transformed RTs outside of the mean $\pm 3 S D$ were removed. We also excluded trials with incorrect responses. Since many studies (Appelle, 1972; Li, Peterson, \& Freeman, 2003; Nasr, \& Tootell, 2012) have shown that human vision is more sensitive to cardinal (horizontal and vertical) than oblique orientations, the adjustment responses were corrected for cardinal biases by fitting a fourth-degree polynomial on distance to cardinal orientations for each participant and taking the residuals (the analysis of uncorrected data yielded the same results). The fourth-degree polynomial was fit using the robust regression procedure ( $\mathrm{rlm}$ function from the MASS package in $R$ ) that fits the model by applying an iteratively reweighted least-squares approach.

The parameters of the adjustment response distributions were then estimated by fitting a mixture of uniform and von Mises distributions (Zhang \& Luck, 2008) to the observers' responses. The fitting was done separately for each observer and each condition included in a particular analysis. For example, for our main analysis of interest, we fitted a mixture of uniform and von Mises distributions for each participant and each combination of target and distractor conditions (i.e., $20 \times 2$ $\times 2$ ). For the analysis of effects of N-2 and N-3 targets, the fitting was done for each participant and each target condition (i.e., $20 \times 2$ for $\mathrm{N}-2$ and $20 \times 2$ for $\mathrm{N}-3$ ), and so on. The mixture of von Mises and uniform was fit using maximum likelihood estimation with ten different starting points for the mixture proportion (from 0.01 to 0.91 in steps of 0.1 ; the starting points for the mean and precision of von Mises were chosen from randomly from $[-\pi, \pi]$ and $[0,10]$ range, respectively). After running ten MLE optimization runs with the aforementioned starting points, the one with the highest likelihood was used in the following analyses. The mean of the von Mises part of the fitted distribution provides information about systematic shifts in target perception while random responses (e.g., from attention lapses) are reflected in the uniform part.

\section{Results}

\section{Judgments of target orientation}

A repeated-measures ANOVA with the mean of the von Mises part of the fitted distribution as the dependent variable was used to study the effects of the previous target and distractor distributions on orientation judgments for the target. ${ }^{1}$ The estimated orientation of the target on the last trial was pushed

\footnotetext{
${ }^{1}$ These analyses were performed on the parameters of fitted distributions rather than the raw data. We therefore present hierarchical analsyses performed on the raw data in supplementary information, which lead to similar results.
}

away from the distractors, $F(1,19)=4.93, p=.039, \eta_{\mathrm{G}}^{2}=.07$ $\left(M=-0.59^{\circ}, S D=2.34^{\circ}\right.$ for $\mathrm{T}<\mathrm{D}$ and $M=0.38^{\circ}, S D=2.37^{\circ}$ for $\mathrm{T}>\mathrm{D})$. In contrast, it was pulled towards the preceding target, $F(1,19)=36.88, p<.001, \eta_{\mathrm{G}}^{2}=.45\left(M=1.45^{\circ}, S D\right.$ $=1.76^{\circ}$ for $\mathrm{T}<$ PrevT and $M=-1.66^{\circ}, S D=1.88^{\circ}$ for $\mathrm{T}>$ PrevT), similar to previously observed serial dependence effects (Figs. 2A and B). Both effects were observed for 19 out of 20 participants as shown in the slopes in Figs. $2 \mathrm{C}$ and D. Interestingly, there was no interaction between the orientation of the previous target and current distractors, $F(1,19)=0.26$, $p=.614, \eta_{\mathrm{G}}^{2}<.01$.

Although our main question involved the distractor and target repetition effects, for completeness we also assessed any effects of the distractor distribution type (Gaussian and uniform) on previous target and distractor distribution effects with a $2 \times 2$ (distractors relative orientation $\times$ distractor distribution type) repeated-measures ANOVA. As in the previous analysis, the mean part of the fitted distribution was used as the dependent variable. The results showed that neither the main effect of distribution type, $F(1,19)=0.05, p=.820$, $\eta_{\mathrm{G}}^{2}<.01$, nor the interaction with the target-distractor relationship, $F(1,19)=4.08, p=.058, \eta_{\mathrm{G}}^{2}=.03$, were significant.

\section{Temporal effects and target and distractor distance}

It is well known that history effects upon visual perception can last for a long time (Brascamp, Pels, \& Kristjánsson, 2011; Fischer \& Whitney, 2014; Maljkovic \& Nakayama, 1994; see review in Kristjánsson \& Ásgeirsson, 2019). For example, Fischer and Whitney (2014) found that their serial dependence effect lasted for at least three trials. We therefore analyzed cumulative effects of the target ${ }^{2}$ (one-back, two-back and three-back targets) during the learning trials after excluding the adjustment responses identified by the mixture model as belonging to a uniform component with probability $>0.5$ ( $8.7 \%$ of trials). Figure 3 shows the results. Similar to the main analyses, the mean parts of the mixture distribution (dependent variable) were estimated for trials where preceding targets were clockwise or counterclockwise relative to the probed target, controlling for the distractor-to-target orientation difference. We found that $\mathrm{N}-2$ targets created a significant bias in adjustment response $(M=1.16[0.60,1.73], t(19.0)=$ $4.32, p<.001)$, which was weaker than the bias created by the immediately preceding target $(M=3.23[2.26,4.19], t(19.0)=$ $7.01, p<.001$ ), while the target on the $\mathrm{N}-3$ trial did not create a significant bias $(M=0.22[-0.56,1.00], t(19.0)=0.59, p=$ $.564)$. We also performed a control analysis using the $\mathrm{N}+1$ target, and, as expected, it did not create any bias $(M=-0.55$ $[-1.17,0.07], t(19.0)=-1.86, p=.078)$.

\footnotetext{
${ }^{2}$ We could not estimate the effect from distractors on previous trials with the current design as distractor mean was kept constant during learning.
} 

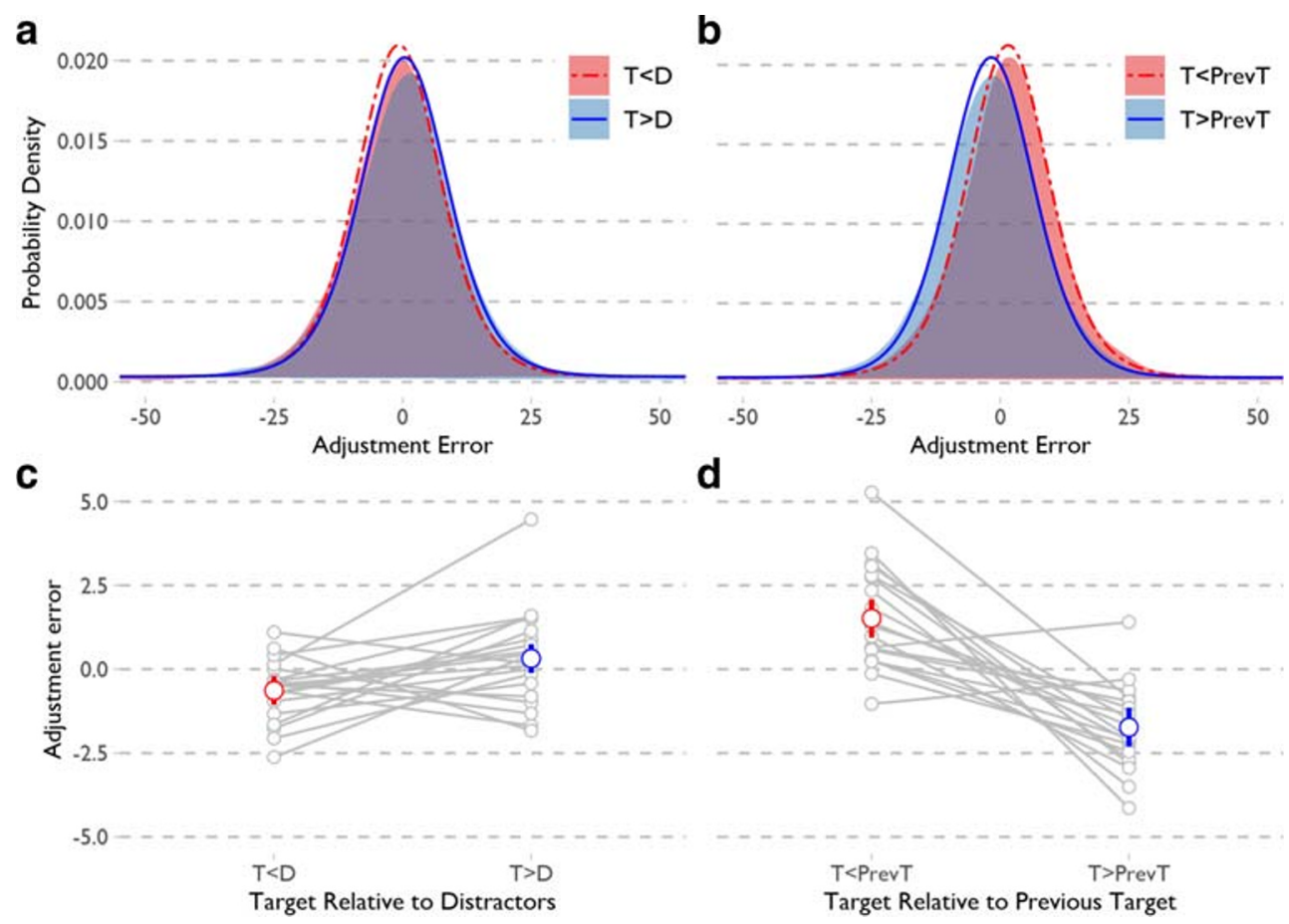

Fig. 2 Effects of preceding distractor distribution and previous target on perceived orientation. (A) and (B): Shaded areas show the raw response error distribution. The lines show the fitted mixture model that combined the Gaussian and uniform distributions. (C) and (D): The mean adjustment error by participants (gray lines) and the average across participants with a 95\% confidence interval (blue and red bars). $\mathrm{T}<\mathrm{D}$

Additionally, we studied the role of the mean distance between the target and distractors in feature space on adjustment error using the same mixed effects approach as described above. The results revealed that the bias away from the

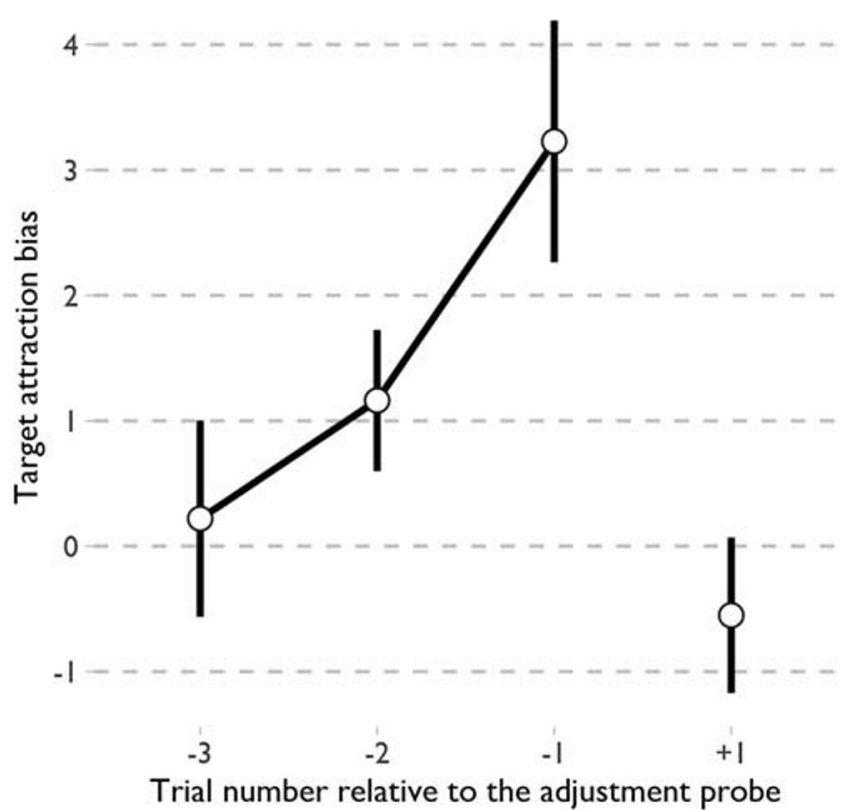

Fig. 3 Attraction bias created by targets in preceding trials and in a control analysis using the next trial. Bars show $95 \%$ confidence intervals means that the target was oriented counterclockwise to distractors, while $\mathrm{T}>\mathrm{D}$ indicates that it was oriented clockwise to distractors. $\mathrm{T}<\mathrm{PrevT}$ and $\mathrm{T}>\mathrm{PrevT}$ indicate the same relative to the previous target. Note that in panels $\mathrm{A}$ and $\mathrm{C}$, the shift in the means of the response probabilities is away from the distractors (repulsion effect), while in panels B and D it is toward the previous target (attraction effect)

distractor mean was similar for different distractors, nonsignificantly increasing with distance between the target and distractors, $B=0.37, S E=2.53, t(14.98)=0.15, p=.886$.

\section{Performance on search trials and the effect of search performance on reported orientation}

We also measured the effects of the different distributions, with RTs and accuracy on the search trials as dependent variables, using repeated-measures ANOVAs. As Fig. 4 shows, visual search accuracy was higher when the distractor distribution was Gaussian than when it was uniform, $F(1,19)=$ 21.62, $p<.001, \eta_{\mathrm{G}}^{2}=.05(M=96.0, S D=2.4$ for Gaussian and $M=94.6, S D=2.4$ for Uniform) and changed over the learning trials, $F(4,76)=4.42, p=.008, \eta_{\mathrm{G}}^{2}=.04$. RTs were also affected by distractor distribution, $F(1,19)=66.81, p<$ $.001, \eta_{\mathrm{G}}^{2}=.02(M=868.5, S D=261.8$ for Gaussian and $M=$ 962.4, $S D=308.1$ for Uniform) and by trial number, $F(4,76)$ $=9.04, p=.002, \eta_{\mathrm{G}}^{2}=.03$. We then ran a polynomial mixed effect regression to assess the effect of trial repetition in more detail. For accuracy, only the linear effect on trial number was significant, $B=22.95, S E=3.47, Z=6.61, p<.001$, while for RT there was a quadratic relationship, $B=5.27, S E=1.31$, $t(19.02)=4.03, p<.001$. This pattern of results suggests that while observers benefitted from repetitions (resulting in decreased RT after the first trial), they also spent more time 
a
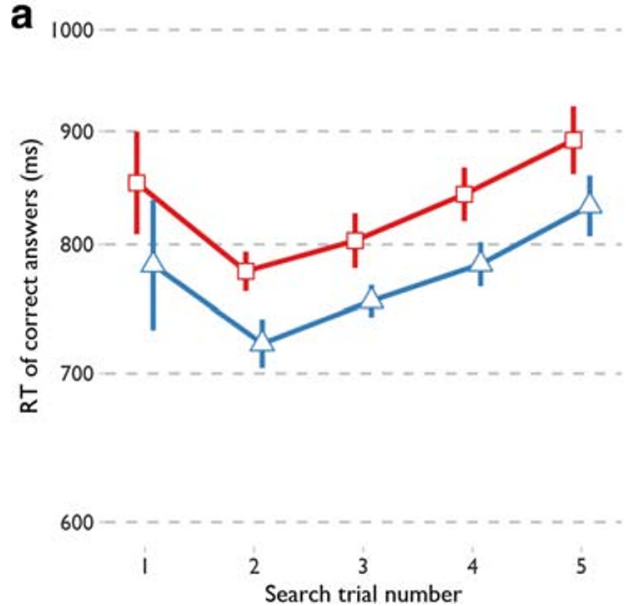

b 100.0

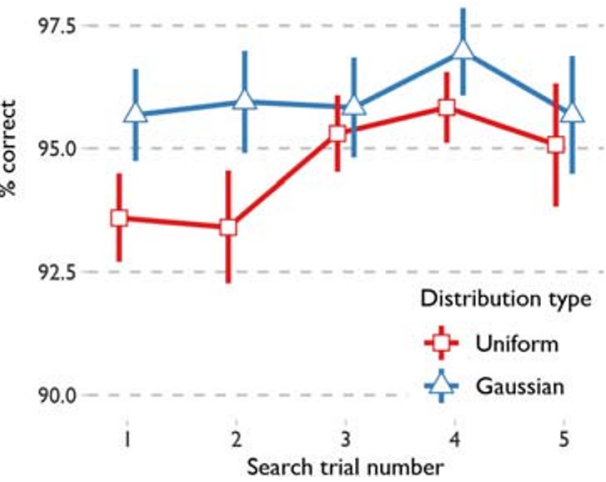

Fig. 4 Performance on the search trials. Response times (RTs) on the left and accuracy on the right. Bars show 95\% confidence intervals

analyzing the stimuli towards the end of the learning trials blocks, possibly preparing for the upcoming adjustment task.

We then analyzed the time course of distractor and target effects using search times on the last search trial and adjustment times. First, we aimed to see if the amount of time spent searching for a target (and, potentially, memorizing it) affects the observed bias magnitude. To account for differences in search time distributions between observers, we used RT percentiles for each observer. For statistical tests, we used a mixed model that included the interaction of RT percentile and the effect of interest. As Fig. 5 shows, the effects of both previous target and distractors were similar across search times $(B=0.87, S E=1.13, t(45.13)=0.77, p=.444$ for the interaction of RT with the previous target effect, and $B=0.47$, $S E=1.18, t(25.75)=0.40, p=.696$ for the interaction with the distractor effect). Similarly, adjustment time did not significantly interact with the effects of interest $(B=0.24, S E=1.04$, $t(130.92)=0.23, p=.820$ for the interaction of RT with the previous target effect, and $B=-1.03, S E=1.10, t(81.40)=-$ $0.93, p=.353$ for the interaction with the distractor effect).

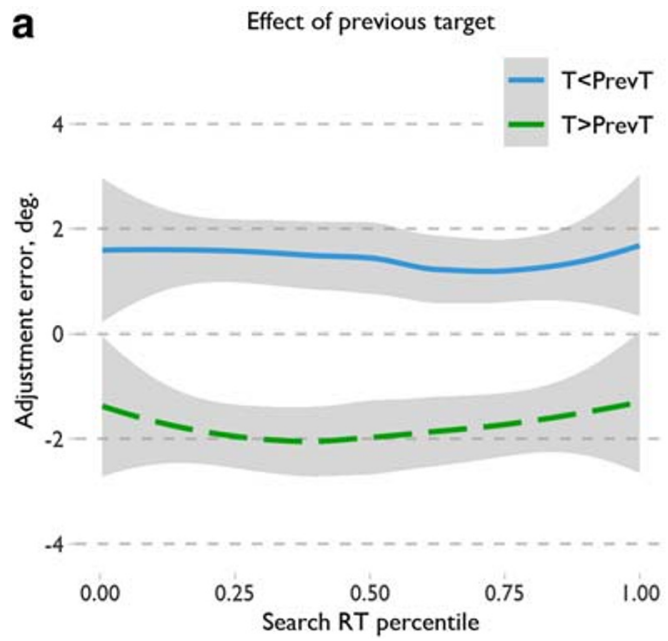

This suggests that the amount of time observers spent searching for the target or memorizing it as well as how deliberate they were in their adjustment responses was not crucial for the biases we observed.

\section{Discussion}

Our results are the first to show that when observers scan their visual environment, two simultaneous biases occur that pull the judgment of the orientation of a line in different directions. Firstly, our results show that orientation judgments of a single line can be biased towards a recently attended target line, consistent with findings on serial dependence (Fischer \& Whitney, 2014). This occurs even though observers did not have to decide, on preceding trials, what the target orientation was. Secondly, we show for the first time that the judgment of the orientation of a line can be biased away from an ensemble of to-be-ignored items, in our case distractors in a visual search task. We propose that these two parallel effects serve b Effect of distractors
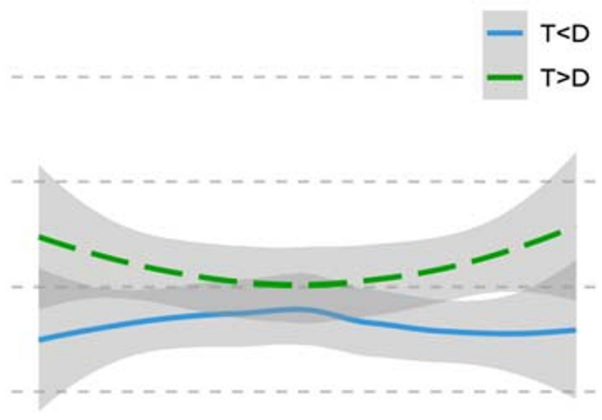

0.00
1.00

$$
0.25 \text { Search RT percentile } 0.75
$$

Fig. 5 The time course of target and distractor effects as a function of search response time (RT). Shaded areas show $95 \%$ confidence intervals 
a similar purpose in reducing noise and preserving continuity in perception, biasing perception towards important stimuli and away from items that should be ignored. Although the effects of previous targets and distracters on target perception were not particularly large, they were stable for the vast majority of participants and such biases can nevertheless affect perception in important ways.

Studies on visual attention have shown that by selecting and limiting the information that is available at different levels of perceptual processing, attention can optimize perception, selecting stimuli of importance (Driver, 2001; Kristjánsson \& Egeth, 2019). Interestingly, serial dependence is observed for attended items but not for unattended ones (Fischer \& Whitney, 2014; Fornaciai \& Park, 2018; Fritsche \& de Lange, 2019; Liberman, Zhang, \& Whitney, 2016). However, ignored items differ categorically from unattended ones. Recent studies suggest that we are remarkably good at picking up intricate patterns over time from ignored stimuli (Chetverikov et al., 2016, 2017b, c; Chetverikov et al., 2019; Hansmann-Roth et al., 2019). The results of the present study further show the importance of ignored items. To-be-ignored objects, in addition to the ones selected by attention filtering systems, can bias perception.

Fischer and Whitney (2014) introduced the concept of the continuity field, which integrates consecutive stimuli to promote perceptual stability and continuity. They argued that serial dependence from targets was due to the operation of this continuity field, which in turn reflects constant attempts of our brain to infer the present based on the past. This is consistent with the behaviour of an optimal observer in a Bayesian framework (Burr \& Cicchini, 2014; Cicchini et al., 2017; Kalm \& Norris, 2018; van Bergen \& Jehee, 2019). However, in preserving continuity, ignored stimuli might be no less important than attended ones. Our results highlight that the continuity field concept will need to encompass effects upon perception from the stimuli that we actively try to ignore.

Our paradigm also opens up the avenue of testing serial dependencies for visual ensembles where ignored and attended items can be contrasted. Manassi et al. (2017) have previously shown serial dependencies for ensembles of oriented Gabor patches, and that this effect occurred at the level where a group of objects was perceived as ensembles, but our results argue that such dependencies may differ depending on the role particular items play within visual ensembles.

In a serial dependence study, the items can be treated as multiple observations coming from the same source. We may, in other words, assume that as in the real world, sequential observations are likely to originate from the same object (Kiyonaga et al., 2017). Under that assumption, it makes sense to merge the incoming sensory inputs to obtain a more precise percept. However, in the visual search task that we tested, targets and distractors clearly do not belong to the same source. This is especially evident in odd-one-out search where the target is defined as the item that stands out, the item that is different from the other items (the distractors). Under the assumption that the target comes from a different source than distractors, a negative bias from distractors might in fact be optimal. Consider the case with categorically defined distractors in an odd-one-out color search. When you know from previous experience that the target is anything but red (a priori, using the ideal observer framework language), and your sensory information tells you that it is between red and yellow (a likelihood), it is likely that the target is actually yellow and the part of the sensory information that suggests red color is just noise. This is similar to the negative biases sometimes observed in multisensory integration with high stimulus disparities that are explained by a causal inference model (Körding et al., 2007; Wallace et al., 2004).

Target representations might also become tuned during retention or decision making to avoid interference from distractor memory. This is in line with previous findings in visual working memory (VWM) studies, where distractors were found to affect target representations as well. However, the specific pattern of results in our study is different. Rademaker, Bloem, De Weerd, and Sack (2015) found that when observers have to remember the first of two sequentially presented stimuli, memory is biased towards the second, irrelevant stimulus. When both stimuli have to be remembered, the bias is severely reduced. This is similar to a positive serial dependence effect. In contrast, Golomb (2015) found that for two simultaneously presented items, the memory of the target is biased away from the distractor when they are similar but towards it when they are dissimilar. Similar results were also reported by Chunharas, Rademaker, Brady, and Serences (2019) for hue memory, but they found that repulsion turns to attraction under high memory load. Bae and Luck (2017) also found that when two sequentially shown motion directions are to be remembered, high similarity leads to repulsive biases but low similarity to attractive ones. These studies suggest that dissimilar distractors, such as the ones used in our odd-oneout search task, should create attractive rather than repulsive biases. Thus, while we cannot reject the possibility that distractors affected target representations, the mechanisms are likely to be different from those observed in VWM studies. It is important to note, however, that the extent to which serial dependence reflects perception and to what degree it reflects VWM is a hotly debated topic that cannot be addressed with the current data (see Kiyonaga et al., 2017 for review).

The observed repulsion bias cannot be explained by wellknown adaptation and simultaneous contrast effects in orientation perception. The tilt illusion and the tilt aftereffect are known to produce repulsive biases in perceived orientation (Gibson, 1937; Gibson \& Radner, 1937). However, these biases occur when the target stimulus is relatively similar to inducers and changed into attractive biases when the two are distinct (see, e.g., Clifford, 2014, for a review). In contrast, we 
did not observe any dependence on target-to-distractor similarity, and in the range of orientation difference we studied $\left(70-90^{\circ}\right)$ both the tilt illusion and tilt aftereffect should create positive rather than negative biases.

\section{Is serial dependence a perceptual or decisional bias?}

Serial dependence in visual search is especially interesting because by itself visual search does not require explicit judgments of target features. Serial dependence studies typically involve sequential decisions on stimulus features, leading some researchers to conclude that serial dependence occurs post-perceptually (Bliss et al., 2017; Fritsche, Mostert, \& de Lange, 2017), while others have argued that perception is affected (Cicchini, Mikellidou, \& Burr, 2017; Fornaciai \& Park, 2018; Gekas, McDermott, \& Mamassian, 2019; Manassi et al., 2018, 2019). Recently, Pascucci et al. (2019) further developed the idea of decisional biases suggesting that even without an explicit task, observers might continuously make implicit decisions about target orientation, applying the same "decisional template." Our findings, however, argue that decision making is not necessary for serial dependence, even in this implicit form. In the odd-one-out search task, on the majority of trials observers need to analyze orientation but only to find a target, not to report the orientation itself, and the target is defined as the item that differs most from the others. It is unlikely that on each trial of the search task observers make an implicit decision about target orientation. Instead, they utilize the perceived orientation of different stimuli to decide which one is most likely to be the target (Ma, Shen, Dziugaite, \& van den Berg, 2015; Schoonveld, Shimozaki, \& Eckstein, 2007). That is, while they have information about target orientation, the "decisional template" involves the target location. Thus, given that we are able to observe serial dependence in the context of a search task, it is likely to be caused by representations of previously presented stimuli rather than by decisions about them. Note, however, that serial dependence may of course arise at many stages of processing (Cicchini \& Kristjánsson, 2015; Kiyonaga et al., 2017).

\section{Accuracy and response times during learning trials}

Before discussing RT and accuracy for our so-called learning trials, we must note that the task was a tool toward assessing history effects upon orientation judgments, rather than being thought of as a measure in this study. The results for performance accuracy (Fig. 4B) are in agreement with previous studies (Chetverikov et al., 2016, 2017b, c, d), suggesting that participants learned the probability distribution of the distractors, resulting in more accurate visual search in the last trials within a block than the first trials. However, the RT analysis (Fig. 4A) showed that RTs did not improve during the learning trials in contrast with the previous studies. A possible explanation for this RT pattern is that since participants did not know how many search trials would occur in each block, after the second trial they may have started to prepare for the later adjustment task, perhaps causing delays in responding.

\section{Conclusions}

Our results show that perception reliably reflects not only what is attended in each case (serial dependence), but for the first time our results reveal serial dependencies from ignored information, in this case distractors in a search task. Importantly, the bias arises even when distractors are very dissimilar to targets, distinguishing it both from the wellknown tilt illusion and the tilt adaptation. Additionally, our results show that explicit reports of stimulus features are not necessary for serial dependence. The results suggest that perception takes both attended and ignored stimuli into account in preserving the continuity of the visual world to an even larger degree than previously found.

Acknowledgements We would like to thank the members of the Icelandic vision lab for taking part in our experiment and for inspiring comments throughout the process. This work was supported by the Icelandic Research Fund (grant \# 173947-052) and the research fund of the University of Iceland. AC is supported by Radboud Excellence Fellowship.

\section{References}

Alais, D., Kong, G., Palmer, C., \& Clifford, C. (2018). Eye gaze direction shows a positive serial dependency. Journal of Vision, 18(4),11 . doi: https://doi.org/10.1167/18.4.11

Alexi, J., Cleary, D., Dommisse, K., Palermo, R., Kloth, N., Burr, D., \& Bell, J. (2018). Past visual experiences weigh in on body size estimation. Scientific Reports, 8(1), 215. doi: https://doi.org/10.1038/ s41598-017-18418-3

Allred, S. R. (2012). Approaching color with Bayesian algorithms. Visual Experience: Sensation, Cognition, and Constancy, 212-231. doi: https://doi.org/10.1093/acprof:oso/9780199597277.003.0012

Appelle, S. (1972). Perception and discrimination as a function of stimulus orientation: the" oblique effect" in man and animals. Psychological bulletin, 78(4), 266.

Arita, J. T., Carlisle, N. B., \& Woodman, G. F. (2012). Templates for rejection: configuring attention to ignore task-irrelevant features. Journal of Experimental Psychology: Human Perception and Performance, 38(3), 580-584. doi: https://doi.org/10.1037/ a0027885

Bae, G.-Y., \& Luck, S. J. (2017). Interactions between visual working memory representations. Attention, Perception, \& Psychophysics, 79(8), 2376-2395. doi: https://doi.org/10.3758/s13414-017-1404-8 
Beck, V. M., \& Hollingworth, A. (2015). Evidence for negative feature guidance in visual search is explained by spatial recoding. Journal of Experimental Psychology: Human Perception and Performance, 41(5), 1190-1196. doi: https://doi.org/10.1037/xhp0000109

Bliss, D. P., Sun, J. J., \& D'Esposito, M. (2017). Serial dependence is absent at the time of perception but increases in visual working memory. Scientific Reports, 7(1), 14739. doi: https://doi.org/10. 1038/s41598-017-15199-7

Brainard, D. H. (1997). The psychophysics toolbox. Spatial Vision, 10(4), 433-436. doi: https://doi.org/10.1163/156856897X00357

Brainard, D. H. (2009) Bayesian approaches to color vision. In: The Cognitive Neurosciences, $4^{\text {th }}$ edition, ed. Gazzaniga, M., pp. 395408. MIT Press.

Brascamp, J. W., Pels, E., \& Kristjánsson, Á. (2011). Priming of pop-out on multiple time scales during visual search. Vision Research, 51(17), 1972-1978. doi: https://doi.org/10.1016/j.visres.2011.07. 007

Burr, D., \& Cicchini, G. M. (2014). Vision: efficient adaptive coding. Current Biology, 24(22), R1096-R1098. doi: https://doi.org/10. 1016/j.cub.2014.10.002

Chelazzi, L., Marini, F., Pascucci, D., \& Turatto, M. (2019). Getting Rid of Visual Distractors: The Why, When, How and Where. Current Opinion in Psychology, 29: 135-147 doi:https://doi.org/10.1016/j. copsyc.2019.02.004.

Chetverikov, A., Campana, G., \& Kristjánsson, A. (2016). Building ensemble representations: How the shape of preceding distractor distributions affects visual search. Cognition, 153, 196-210. doi: https://doi.org/10.1016/j.cognition.2016.04.018

Chetverikov, A., Campana, G., \& Kristjánsson, A. (2017a). Learning features in a complex and changing environment: A distributionbased framework for visual attention and vision in general. Progress in Brain Research, 236, 97-120. doi: https://doi.org/10. 1016/bs.pbr.2017.07.001

Chetverikov, A., Campana, G., \& Kristjánsson, A. (2017b). Rapid learning of visual ensembles. Journal of Vision, 17(2), 21. doi: https://doi. org/10.1167/17.2.21

Chetverikov, A., Campana, G., \& Kristjánsson, A. (2017c). Representing Color Ensembles. Psychological Science, 28(10), 1510-1517. doi: https://doi.org/10.1177/0956797617713787

Chetverikov, A., Campana, G., \& Kristjánsson, A. (2017d). Set size manipulations reveal the boundary conditions of perceptual ensemble learning. Vision Research, 140, 144-156. doi: https://doi.org/10. 1016/j.visres.2017.08.003

Chetverikov, A., Campana, G., \& Kristjánsson, Á. (2020). Probabilistic rejection templates in visual working memory. Cognition, 196, 104075, https://doi.org/10.1016/j.cognition.2019.104075

Chetverikov, A., Hansmann-Roth, S., Tanrikulu, O. D., \& Kristjánsson, Á. (2019). Feature distribution learning (FDL): A new method to study visual ensembles with priming of attention shifts. In Pollman S. (Ed). Spatial learning and attention guidance. New York, NY: Neuromethods, Springer Nature. 1-21. doi: https://doi.org/10.1007/ 7657201920

Chunharas, C., Rademaker, R. L., Brady, T. F., \& Serences, J. (2019, February 4). Adaptive memory distortion in visual working memory. Preprint is available at PsyArXiv: 10.31234/osf.io/e3m5a

Cicchini, G. M., \& Kristjánsson, Á. (2015). Guest editorial: On the possibility of a unifying framework for serial dependencies. i. Perception, 6(6).

Cicchini, G. M., Mikellidou, K., \& Burr, D. (2017). Serial dependencies act directly on perception. Journal of Vision, 17(14), 6. doi: https:// doi.org/10.1167/17.14.6

Clifford, C. W. (2014). The tilt illusion: phenomenology and functional implications. Vision Research, 104, 3-11. doi: https://doi.org/10. 1016/j.visres.2014.06.009

Cunningham, C. A., \& Egeth, H. E. (2016). Taming the White Bear: Initial Costs and Eventual Benefits of Distractor Inhibition.
Psychological Science, 27(4), 476-485. doi: https://doi.org/10. $1177 / 0956797615626564$

Driver, J. (2001). A selective review of selective attention research from the past century. British Journal of Psychology, 92(1), 53-78. doi: https://doi.org/10.1348/000712601162103

Fischer, J., \& Whitney, D. (2014). Serial dependence in visual perception. Nature Neuroscience, 17(5), 738-743. doi: https://doi.org/10.1038/ nn.3689

Fornaciai, M., \& Park, J. (2018). Serial dependence in numerosity perception. Journal of Vision, 18(9), 15. doi: https://doi.org/10.1167/18. 9.15

Fritsche, M., \& de Lange, F. P. (2019). The role of feature-based attention in visual serial dependence. bioRxiv, 584789. https://doi.org/10. $1101 / 584789$

Fritsche, M., Mostert, P., \& de Lange, F. P. (2017). Opposite Effects of Recent History on Perception and Decision. Current Biology, 27(4), 590-595. doi: https://doi.org/10.1016/j.cub.2017.01.006

Gaspelin, N., \& Luck, S. J. (2018). The Role of Inhibition in Avoiding Distraction by Salient Stimuli. Trends in Cognitive Sciences, 22(1): 79-92. doi:https://doi.org/10.1016/j.tics.2017.11.001

Gekas, N., McDermott, K. C., \& Mamassian, P. (2019). Disambiguating serial effects of multiple timescales. Journal of Vision, 19(6), 24. doi: https://doi.org/10.1167/19.6.24

Geng, J. J., DiQuattro, N. E., \& Helm, J. (2017). Distractor probability changes the shape of the attentional template. Journal of Experimental Psychology: Human Perception and Performance, 43(12), 1993-2007. doi: https://doi.org/10.1037/xhp0000430

Geng, J. J., \& Witkowski, P. (2019). Template-to-distractor distinctiveness regulates visual search efficiency. Current Opinion in Psychology, 29, 119-125. doi: https://doi.org/10.1016/j.copsyc. 2019.01.003

Geng, J. J., Won, B. Y., \& Carlisle, N. B. (2019). Distractor ignoring: Strategies, learning, and passive filtering. Current Directions in Psychological Science, 28: 600-606. https://doi.org/10.1177/ 0963721419867099

Gibson, J. J. (1937). Adaptation, after-effect, and contrast in the perception of tilted lines. II. Simultaneous contrast and the areal restriction of the after-effect. Journal of Experimental Psychology, 20(6), 553569.

Gibson, J. J., \& Radner, M. (1937). Adaptation, after-effect and contrast in the perception of tilted lines. I. Quantitative studies. Journal of Experimental Psychology, 20(5), 453-467.

Girshick, A. R., Landy, M. S., \& Simoncelli, E. P. (2011). Cardinal rules: visual orientation perception reflects knowledge of environmental statistics. Nature Neuroscience, 14(7), 926-932. doi: https://doi.org/ 10.1038/nn.2831.

Golomb, J. D. (2015). Divided spatial attention and feature-mixing errors. Attention, Perception, \& Psychophysics, 77(8), 2562-2569. doi: https://doi.org/10.3758/s13414-015-0951-0

Hansmann-Roth, S., Chetverikov, A., \& Kristjánsson, A. (2019). Representing color and orientation ensembles: Can observers learn multiple feature distributions? Journal of Vision, 19(9), 2. doi: https://doi.org/10.1167/19.9.2

Kalm, K., \& Norris, D. (2018). Visual recency bias is explained by a mixture model of internal representations. Journal of Vision, 18(7): 1. doi: https://doi.org/10.1167/18.7.1

Kiyonaga, A., Scimeca, J. M., Bliss, D. P., \& Whitney, D. (2017). Serial Dependence across Perception, Attention, and Memory. Trends in Cognitive Sciences, 21(7), 493-497. doi: https://doi.org/10.1016/j. tics.2017.04.011

Kleiner, M., Brainard, D., \& Pelli, D. (2007). What's new in Psychtoolbox-3? Perception, 36(14):1-16

Körding, K. P., Beierholm, U., Ma, W. J., Quartz, S., Tenenbaum, J. B., \& Shams, L. (2007). Causal inference in multisensory perception. PLoS One, 2(9), e943. doi: https://doi.org/10.1371/journal.pone. 0000943 
Kristjánsson, Á., \& Ásgeirsson, Á. G. (2019). Attentional priming: recent insights and current controversies. Current Opinion in Psychology, 29, 71-75.

Kristjánsson, Á., \& Egeth, H. (2019). How feature integration theory integrated cognitive psychology, neurophysiology, and psychophysics. Attention, Perception, \& Psychophysics, 1-17. doi: https://doi. org/10.3758/s13414-019-01803-7.

Li, B., Peterson, M. R., \& Freeman, R. D. (2003). Oblique effect: a neural basis in the visual cortex. Journal of neurophysiology, 90(1), 204217.

Liberman, A., Zhang, K., \& Whitney, D. (2016). Serial dependence promotes object stability during occlusion. Journal of Vision, 16(15), 16. doi: https://doi.org/10.1167/16.15.16

Ma, W. J., Shen, S., Dziugaite, G., \& van den Berg, R. (2015). Requiem for the max rule? Vision Research, 116, 179-193. doi: https://doi.org/ 10.1016/j.visres.2014.12.019

Maljkovic, V., \& Nakayama, K. (1994). Priming of pop-out: I. Role of features. Memory \& Cognition, 22(6), 657-672. doi: https://doi.org/ 10.3758/BF03209251

Manassi, M., Kristjánsson, Á., \& Whitney, D. (2019). Serial dependence in a simulated clinical visual search task. Scientific Reports, 9(1), 110.

Manassi, M., Liberman, A., Chaney, W., \& Whitney, D. (2017). The perceived stability of scenes: serial dependence in ensemble representations. Scientific Reports, 7(1), 1-9.

Manassi, M., Liberman, A., Kosovicheva, A., Zhang, K., \& Whitney, D. (2018). Serial dependence in position occurs at the time of perception. Psychonomic Bulletin \& Review, 25(6), 2245-2253. doi: https:// doi.org/10.3758/s13423-018-1454-5

Nasr, S., \& Tootell, R. B. (2012). A cardinal orientation bias in sceneselective visual cortex. Journal of Neuroscience, 32(43), 1492114926.

Noonan, M. P., Crittenden, B. M., Jensen, O., \& Stokes, M. G. (2018). Selective inhibition of distracting input. Behavioral Brain Research, 355: 36-47. doi:https://doi.org/10.1016/j.bbr.2017.10.010.

Pascucci, D., Mancuso, G., Santandrea, E., Della Libera, C., Plomp, G., \& Chelazzi, L. (2019). Laws of concatenated perception: Vision goes for novelty, decisions for perseverance. PLOS Biology, 17(3), e3000144. doi: https://doi.org/10.1371/journal.pbio.3000144

Rademaker, R. L., Bloem, I. M., De Weerd, P., \& Sack, A. T. (2015). The impact of interference on short-term memory for visual orientation. Journal of Experimental Psychology: Human Perception and Performance, 41(6), 1650-1665. doi: https://doi.org/10.1037/ xhp0000110

Schoonveld, W., Shimozaki, S. S., \& Eckstein, M. P. (2007). Optimal observer model of single-fixation oddity search predicts a shallow set-size function. Journal of Vision, 7(10),1. doi: https://doi.org/10. $1167 / 7.10 .1$

Simonsohn, U., \& Loewenstein, G. (2006). Mistake: The Effect of Previously Encountered Prices on Current Housing Demand. The
Economic Journal, 116(508), 175-199. doi: https://doi.org/10.1111/ j.1468-0297.2006.01052.x

Simonson, I., \& Tversky, A. (1992). Choice in context: Tradeoff contrast and extremeness aversion. Journal of Marketing Research, 29(3), 281-295. doi: https://doi.org/10.2307/3172740

Sotiropoulos, G., Seitz, A. R., \& Seriès, P. (2011). Changing expectations about speed alters perceived motion direction. Current Biology, 21(21), R883-R884. doi: https://doi.org/10.1016/j.cub.2011.09.013

Suarez-Pinilla, M., Seth, A. K., \& Roseboom, W. (2018). Serial dependence in the perception of visual variance. Journal of Vision, 18(7), 4. doi: https://doi.org/10.1167/18.7.4

Van Bergen, R. S., \& Jehee, J. F. (2019). Probabilistic representation in human visual cortex reflects uncertainty in serial decisions. the Journal of Neuroscience, 3212-18. doi: https://doi.org/10.1523/ JNEUROSCI.3212-18.2019

Wallace, M. T., Roberson, G., Hairston, W. D., Stein, B. E., Vaughan, J. W., \& Schirillo, J. A. (2004). Unifying multisensory signals across time and space. Experimental Brain Research, 158(2), 252-258. doi: https://doi.org/10.1007/s00221-004-1899-9

Wei, X.X., \& Stocker, A. A. (2017). Lawful relation between perceptual bias and discriminability. Proceedings of the National Academy of Sciences, 114(38), 10244-10249. doi: https://doi.org/10.1073/pnas. 1619153114

Weiss, Y., Simoncelli, E. P., \& Adelson, E. H. (2002). Motion illusions as optimal percepts. Nature Neuroscience, 5(6), 598. doi: https://doi. org/10.1038/nn858

Won, B. Y., \& Geng, J. J. (2018). Learned suppression for multiple distractors in visual search. Journal of Experimental Psychology: Human Perception and Performance, 44(7), 1128-1141. doi: https://doi.org/10.1037/xhp0000521

$\mathrm{Yu}, \mathrm{X}$., \& Geng, J. J. (2019). The attentional template is shifted and asymmetrically sharpened by distractor context. Journal of Experimental Psychology: Human Perception and Performance, 45(3): 336-353. doi: https://doi.org/10.1037/xhp0000609

Zhang, W., \& Luck, S. J. (2008). Discrete fixed-resolution representations in visual working memory. Nature, 453(7192), 233-235. doi: https:// doi.org/10.1038/nature06860

Open Practices Statement A preprint of this paper is available at https:// psyarxiv.com $/ \mathrm{m} 79$ nu. The data from the experiment reported in this paper and scripts for simulations and analyses are available at https://osf.io/ zgkn9/.

Publisher's note Springer Nature remains neutral with regard to jurisdictional claims in published maps and institutional affiliations. 